\title{
Availability of Medical Oxygen and its Clinical Practice in Thirty Two Public Hospitals of Ethiopia: A Cross-Sectional Time-Series Design
}

Habtamu Seyoum Tolla ( $\sim$ hseyoum@clintonhealthaccess.org )

Clinton Health Access Initiative https://orcid.org/0000-0003-3549-3397

Yigeremu Abebe Asemere

Clinton Health Access Initiative

Alebel Yaregal Desale

Clinton Health Access Initiative

Dinkineh Bikila Woyessa

Clinton Health Access Initiative

\section{Zinabie Feleke Fekadu}

Clinton Health Access Initiative

Alemayehu Berhanu Belete

Clinton Health Access Initiative

\section{Audrey Battu}

Clinton Health Access Initiative

Felix Lam

Clinton Health Access Initiative

\section{Research article}

Keywords: availability of medical oxygen, functional oxygen, pulse oximetry, in-patient pediatrics

Posted Date: January 29th, 2021

DOl: https://doi.org/10.21203/rs.3.rs-154461/v1

License: (1) This work is licensed under a Creative Commons Attribution 4.0 International License. Read Full License 


\section{Abstract}

Background: Oxygen is an essential medicine that saves many children dying of hypoxemia from pneumonia. Nearly more than $95 \%$ of deaths occur in developing countries. Similarly, the prevalence of pneumonia is high in Ethiopia. Oxygen therapy is a lifesaving treatment, however, the appropriate use and administration of oxygen in clinical practice is often challenging without knowledge of its potential risks and benefits. Therefore, the study aims to assess the functional availability of oxygen devices and its clinical practice in public hospitals of Ethiopia.

Methods: A facility-based cross-sectional time-series design was conducted from December 2015 to December 2019. Primary data was collected from 32 public hospitals bi-annually. Similarly, medical record charts were reviewed retrospectively biannually using interviewer-administered structured questionnaires. A chi-square test that claimed $\mathrm{P}<0.05$ used to assess the statistical significance differences.

Results: The study was conducted in thirty-two public hospitals of Ethiopia, where capacity building and technical support interventions implemented. Of which, 15 (46.9\%) were General hospitals, and the remaining $10(31.2 \%)$ and 7 (21.9\%) were Referral and Primary hospitals respectively. Multifaceted approaches such as on-site and off-site capacity building of healthcare workers, regular mentorship, technical assistance, development of guideline/manuals, oxygen device procurement and maintenance were provided to healthcare workers and health facilities. Functional availability of oxygen has shown a statistically significant increase from $62 \%$ to $100 \%$ in Pediatric In-patient Department of general and referral hospitals ( $p$-value $<0.001$ ). Similarly, functional availability of Pulse Oximetry has shown statistically significant increased from $45 \%$ to $96 \%$. With regard to the clinical practice, SpO2 measurement at diagnosis and admission has increased from $10.2 \%$ to $75 \%$ and $20.5 \%$ to $83 \%$ respectively.

Conclusions: Based on the intervention results, we can conclude that multifaceted approaches targeting healthcare workers capacity, increased device procurement and on-site device maintenance with on-site mentorship can improve the availability of medical oxygen and pulse oximetry and clinical practice of oxygen therapy in health facilities. Therefore, ensuring device availability along with regular technical support and close follow-up of healthcare workers and facilities are key and the initiative needs to be scaled-up.

\section{Background}

Oxygen is an essential medical therapy that saves the lives of many children(1). Oxygen therapy is not only used for pneumonia but also for many other diseases including those which result in hypoxemia (2). Pneumonia is the largest infectious cause of death in children worldwide than any other single infectious disease claiming the lives of an estimated 800,000 children under the age of five, which is equivalent to around 2,200 each day(3) (4). Moreover, COVID-19 could also add 1.9 million to the death tolls this year; 
which could increase all-cause pneumonia deaths by more than $75 \%$. It is further aggravated by the disruption of healthcare services which adds an estimated death of 2.3 million of which $35 \%$ from pneumonia and newborn sepsis (5).

Pneumonia affects developing countries disproportionately as more than $95 \%$ of the deaths occur in developing countries (6) (7). Similarly, the prevalence of pneumonia is high in Ethiopia. A recent study indicated that its prevalence is 33.5 percent(8). Studies suggested that mortality due to childhood pneumonia is strongly linked to poverty-related factors such as malnutrition, lack of safe drinking water and sanitation, indoor air pollution and inadequate access to health care (9) (8).

Hypoxemia, a low level of oxygen in the blood, is a common and frequent complication of pneumonia(6) (10). It is fatal if it is not treated on time(11). Despite the ongoing incongruities, hypoxemia can be detected using clinical assessments or using pulse oximetry since the latter allows simple, non-invasive, and reasonably accurate estimation of arterial oxygen saturation (12). Lack of oxygen leads to dysfunction of the organ system very quickly and then results in imminent death. Hence, hypoxemia is a life-threatening condition that demands early detection and treatment (7).

Hypoxemia coupled with poor clinical conditions, presence of co-morbidities such as malnutrition, incomplete immunization, and socioeconomic factors is a major risk factor for death (7) (10) (13). A systematic review estimated that $13.3 \%$ of children with pneumonia have hypoxemia (14). Furthermore, the UN IGME report indicated that out of 5.9 million annual child deaths 23 percent related to neonatal conditions such as birth asphyxia, sepsis, and low birth weight all of which can lead to hypoxemia (7). Therefore, the diagnosis of hypoxemia and administration of oxygen are key strategies in reducing pneumonia-related mortality among children under the age of five(15). A clinical review update also reported that oxygen therapy is a lifesaving treatment, however, the use and administration of oxygen in clinical practice is often inappropriate without knowledge of its potential risks and benefits (16). This is further supported by the findings of a review on prescription and delivery practice, which reported that although there are some changes in oxygen prescription practice there remains a significant room for improvement (17). Therefore, the study aims to assess the functional availability of oxygen devices and clinical practices of oxygen therapy in in-patient pediatrics department from public hospitals of Ethiopia.

\section{Methods}

\section{Study design, setting and population}

The study employed a facility-based cross-sectional time-series design. The study setting was thirty-two functional public hospitals located in nine regions and two city administrations. These hospitals are selected as model sites for training, mentorship and ongoing data collection in consultation with Ministry of Health and the respective Regional Health Bureaus (RHBs). The selected public hospitals were considered as the study population. Health Care Workers (HCWs) working in the selected public hospitals were also part of the study population since interventions focusing on knowledge and skill on oxygen 
therapy were provided to them. Moreover, medical record reviews of children 0-59 months of age with severe pneumonia cases were the study population.

\section{Sampling and sample size}

Thirty-two functional public hospitals were purposefully selected as model site across nine regions and two city administrations. Functional availability of oxygen devices and pulse oximetry assessed across each department in general and pediatric departments in particular. To assess the status of medical oxygen therapy in the pediatric ward, chart reviews were conducted every six months. All under-five children charts reviewed retrospectively and charts with severe pneumonia cases selected. Out of the identified severe pneumonia cases 10 cards were randomly selected in each hospital and this brings the total charts reviewed to 320 . Thereafter, the procedures and applications of oxygen therapy were assessed such as oxygen check-up, saturation records, oxygen prescription if hypoxemic, routes of administration, follow-up status and outcomes of the child. Finally, based on the findings of the chart review technical support was provided to HCWs.

\section{Interventions/exposures}

\section{Capacity building}

To improve access, utilization and practice of oxygen therapy in health facilities both onsite and offsite trainings were provided for healthcare professionals working in public health facilities. Clinton Health Access Initiative (CHAl) in collaboration with Ministry of Health $(\mathrm{MOH})$ and Regional Health Bureaus (RHBs), oxygen therapy for clinician training of trainer (ToT), basic training and orientation had been provided for a total of 2,714 participants out of which 326 biomedical engineers/technicians were trained on oxygen device maintenance and the rest 2,388 clinicians (nurses and doctors) were trained and got orientation on medical oxygen therapy (18). On top of the offsite training, on-job trainings were also provided to health care workers and biomedical engineers/technicians.

\section{Technical support to $\mathrm{MOH}$ and RHBs}

To accelerate the implementation of oxygen and enhance its availability, technical supports were made to $\mathrm{MoH}$ and RHBs. Some of the major technical supports were the development of a training manual on maintenance of oxygen device/POx for Biomedical Engineers/Technicians (BME/Ts), clinician's training material for oxygen and pulse oximetry use, specification of oxygen concentrator, pulse oximetry, oxygen analyzer, and oxygen plant, oxygen quality assessment conducted in 53 hospitals and oxygen and pulse oximetry scale-up road, related to the road map massive advocacy work has been conducted, national taskforce established for the roll-out of road map and mid-term review conducted. 


\section{Supportive supervision/mentorship}

Supportive supervisions were conducted biannually in 32 public hospitals comprised of each tier across all regions of Ethiopia. During the supportive supervision, technical supports were provided to health workers across the pediatric department. Moreover, to assess the functionality of oxygen devices physical observation was also conducted. Based on the technical support and observation, feedback was given to health workers in the presence of the medical director.

\section{Oxygen device support}

To enhance the availability and quality of service provision, oxygen devices such as oxygen concentrator, oxygen analyzer and pulse oximetry were provided. Moreover, as part of on the job training and support, maintenance supports were also provided to biomedical engineers/technicians.

\section{Chart review}

To assess the status of medical oxygen therapy in the pediatric ward, chart reviews were conducted every six months. All under-five children charts reviewed retrospectively and charts with pneumonia cases selected. Out of the identified pneumonia cases 10 cards were randomly selected in each hospital. Thereafter, the procedures and applications of oxygen therapy were assessed such as oxygen check-up, saturation records, oxygen prescription if hypoxemic, routes of administration, follow-up status and outcomes of the child. Based on the findings of the chart review technical support and feedback were provided to the health workers. A framework that illustrates the array of interventions contribute to improve health outcomes of pediatric pneumonia are presented in Figure 1.

\section{Data collection and management}

Structured supportive supervision guidance and checklist were developed to follow-up and assess the variables of interest over the course of time. An additional structured questionnaire file shows this in more detail [see Additional file 1]. Then, the draft checklist shared and reviewed by the regional teams and partners. After incorporation of comments, the final tool was programmed into an electronic data collection tool named SurveyCTO and uploaded onto a server. Then the tool was downloaded into individual tablets and pretested for functionality and user-friendliness. After the tool was finalized, two days' training was provided for the supervisory teams to ensure familiarity with the tool, incorporation of unforeseen issues and minimize biases. Moreover, before the actual implementation of the tool practical demonstration session was also conducted in non-supervision facilities to avoid contamination of information. Finally, the development of the checklist is cognizant of and in consideration to frame a twoway discussion between the supervisors and the health worker at each institution. 
The supportive supervisory teams were composed of CHAl Ethiopia regional uptake officers, RHBs and Ethiopian Pharmaceutical Supply Agency (EPSA) regional team. The supervisors are responsible for conducting the supervisions and providing mentorship and guidance. To ensure the adequacy of mentorship and collect quality data, they had a minimum of first-degree qualification in health and related disciplines and vast experience of working at each tier of the health system. In addition to that, they are well experienced in supervision techniques. The supervisory team provides adequate technical support based on the checklist and record the findings and work with the staff and management of the health facility to bring about improvements on the identified problems. At the end of the technical support, they provide feedback; the team also develops action points and record minutes for mutual close follow-up and implementation.

\section{Data cleaning and analysis}

Data were collected electronically using SurveyCTO platforms and cleaned using MS-Excel Office 2016. Further cleaning and preparation were also made using Statistical Packages for Social Sciences (SPSS). Afterwards, in addition to the existing variables, new variables on the availability of oxygen devices (either oxygen cylinder or concentrator) were created by either/or functions to assess the availability. A descriptive analysis was performed to generate frequencies, tables and charts to present key findings. A chi-square test was used to assess the statistical differences between the baseline and consecutive follow-up supports. A statistical significance relationship was also claimed at $p<0.05$.

\section{Results}

The study conducted in thirty-two public hospitals of Ethiopia, where capacity building and technical support interventions were in effect. Of which, 15 (46.9\%) were General hospitals, and the remaining 10 $(31.2 \%)$ and 7 (21.9\%) were Referral and Primary hospitals respectively (Table 1). According to the health system tier of Ethiopia, these public hospitals serve an estimated catchment population of 51 Million. 
Table 1

Types of hospitals by region

\begin{tabular}{|c|c|c|c|c|}
\hline \multirow[t]{2}{*}{ Region } & \multicolumn{4}{|c|}{ Types of hospital } \\
\hline & Primary & General & Referral & Total \\
\hline Amhara & 2 & 2 & 2 & 6 \\
\hline Oromia & 2 & 2 & 2 & 6 \\
\hline SNNPR & 1 & 3 & 2 & 6 \\
\hline Tigray & 2 & 2 & 2 & 6 \\
\hline Others & 0 & 6 & 2 & 8 \\
\hline Total & $7(21.9 \%)$ & $15(46.9 \%)$ & $10(31.2 \%)$ & $32(100 \%)$ \\
\hline
\end{tabular}

To assess the application of oxygen therapy in the selected public hospitals' under-five medical records were reviewed retrospectively every six months. In terms of the composition of charts, of 320 medical records of children diagnosed with severe pneumonia and admitted to PIPD, $47 \%, 31 \%$, and $22 \%$ were from general, referral, and primary hospitals respectively (Table 2).

Table 2

Medical records by region and types of hospitals

\begin{tabular}{|c|c|c|c|c|}
\hline \multirow[t]{2}{*}{ Region } & \multicolumn{4}{|c|}{ Types of hospital } \\
\hline & Primary & General & Referral & Total \\
\hline Amhara & 20 & 20 & 20 & 60 \\
\hline Oromia & 20 & 20 & 20 & 60 \\
\hline SNNPR & 10 & 30 & 20 & 60 \\
\hline Tigray & 20 & 20 & 20 & 60 \\
\hline Others & 0 & 60 & 20 & 80 \\
\hline Total & $70(22 \%)$ & $150(47 \%)$ & $100(31 \%)$ & $320(100 \%)$ \\
\hline
\end{tabular}

As part of the initiative to increase access and availability of oxygen, onsite and offsite trainings were provided to Biomedical Engineers/Technicians (BME/Ts). In terms of trained BME/Ts 30 (94\%) of the hospitals have trained BME/Ts. Looking into disaggregated data, primary hospitals have a maximum of one trained BME/Ts whereas, at general hospitals, there were up to nine trained BME/Ts. Overall, the highest number of trained BME/Ts were found in general hospitals followed by referral hospitals (Fig. 2).

Availability of functional oxygen cylinder or concentrator in different departments 
As presented in Table 3, overall the availability of functional oxygen was $100 \%$ at Newborn Intensive Care Unit (NICU) across each type of hospital. Similarly, the availability of functional oxygen was $100 \%$ at Pediatric Inpatient Departments (PIPD) of general and referral hospitals on the day of the visit. In general, functional availability of oxygen has shown a statistically significant increase from $62 \%$ at baseline (December 2015)(19) to 100\% in Pediatric IPD of general and referral hospitals ( $p$-value $<0.001$ ).

Table 3

Availability of functional oxygen by department and types of hospitals

\begin{tabular}{|c|c|c|c|c|c|c|c|}
\hline \multirow[t]{3}{*}{ Department } & \multirow[t]{3}{*}{ Responses } & \multicolumn{6}{|c|}{ Types of hospital } \\
\hline & & \multicolumn{2}{|c|}{ Primary } & \multicolumn{2}{|c|}{ General + Referral } & \multicolumn{2}{|c|}{ Total } \\
\hline & & Freq. & $\%$ & Freq. & $\%$ & Freq. & $\%$ \\
\hline \multirow[t]{3}{*}{ Pediatric IPD } & Yes & 7 & $100 \%$ & 25 & $100 \%$ & 32 & $100 \%$ \\
\hline & No & 0 & $0 \%$ & 0 & $0 \%$ & 0 & $0 \%$ \\
\hline & Total & 7 & $100 \%$ & 25 & $100 \%$ & 32 & $100 \%$ \\
\hline \multirow[t]{3}{*}{ Pediatrics Emergency } & Yes & 1 & $100 \%$ & 17 & $89 \%$ & 18 & $90 \%$ \\
\hline & No & 0 & $0 \%$ & 2 & $11 \%$ & 2 & $10 \%$ \\
\hline & Total & 1 & $100 \%$ & 19 & $100 \%$ & 20 & $100 \%$ \\
\hline \multirow[t]{2}{*}{$\mathrm{NICU}$} & Yes & 7 & $100 \%$ & 25 & $100 \%$ & 32 & $100 \%$ \\
\hline & Total & 7 & $100 \%$ & 25 & $100 \%$ & 32 & $100 \%$ \\
\hline
\end{tabular}

As exemplified in Fig. 3, the availability of functional oxygen revealed an increasing trend since baseline and keeps constantly $100 \%$ since the 3rd round Supportive Supervision (SS).

As shown in Table 4, the availability of functional pulse oximetry was $96 \%$ at PIPD of general and referral hospitals on the day of the visit. Overall, the functional availability of POx has shown statistically significant increased from 45\% at baseline (December 2015)(19) to $96 \%$ (December 2019) in pediatric IPD of general and referral hospitals ( $p$-value $<0.001)$. 
Table 4

Availability of functional pulse oximetry by department and types of hospital

\begin{tabular}{|c|c|c|c|c|c|c|c|}
\hline \multirow[t]{3}{*}{ Department } & \multirow[t]{3}{*}{ Responses } & \multicolumn{6}{|c|}{ Types of hospital } \\
\hline & & \multicolumn{2}{|c|}{ Primary } & \multicolumn{2}{|c|}{ General + Referral } & \multicolumn{2}{|l|}{ Total } \\
\hline & & Freq. & $\%$ & Freq. & $\%$ & Freq. & $\%$ \\
\hline \multirow[t]{3}{*}{ Pediatric IPD } & Yes & 6 & $86 \%$ & 24 & $96 \%$ & 30 & $94 \%$ \\
\hline & No & 1 & $14 \%$ & 1 & $4 \%$ & 2 & $6 \%$ \\
\hline & Total & 7 & $100 \%$ & 25 & $100 \%$ & 32 & $100 \%$ \\
\hline \multirow[t]{3}{*}{ Pediatrics Emergency } & Yes & 1 & $100 \%$ & 18 & $94.7 \%$ & 19 & $95 \%$ \\
\hline & No & 0 & $0 \%$ & 1 & $5.3 \%$ & 1 & $5 \%$ \\
\hline & Total & 1 & $100 \%$ & 19 & $100 \%$ & 20 & $100 \%$ \\
\hline \multirow[t]{3}{*}{$\mathrm{NICU}$} & Yes & 6 & $86 \%$ & 24 & $100 \%$ & 30 & $97 \%$ \\
\hline & No & 1 & $14 \%$ & 0 & $0 \%$ & 1 & $3 \%$ \\
\hline & Total & 7 & $100 \%$ & 24 & $100 \%$ & 31 & $100 \%$ \\
\hline
\end{tabular}

\section{Clinical practices of oxygen therapy}

To assess the application of oxygen therapy in the selected public hospitals' under-five medical records were reviewed retrospectively every six months. Considering the status of POx application at diagnosis and any point during admission of 320 medical record review, only 241 (75\%) and 266 (83\%) of the children had oxygen saturation measurements using POx at diagnosis and any point after diagnosis respectively (Fig. 4). As displayed in the graph below, SpO2 measurement at diagnosis has sharply increased from 10.2\% (April 2017) to 75\% (December 2019). Similarly, Sp02 measurement at admission also showed progressive increment from $20.5-83 \%$. The increments also depicted statistically significant difference both at diagnosis and admission ( $p$-value < 0.001), Fig. 4.

Out of the medical records of 272 children assessed with pulse oximetry at diagnosis or any point during admission, the majority $210(77 \%)$ were diagnosed with hypoxemia $(\mathrm{SpO} 2<90)$. Of the children with hypoxemia (210), the majority 191 (91\%) were prescribed oxygen at any time (including at diagnosis) during their stay in PIPD/pediatric emergency. The result was also statistically significant ( $p$-value< $0.001)$.

Although there are a sizeable number of hospitals in which the prescriptions did not state flow rate there were progressive improvements from the preceding supportive supervisions with a statistically significant increase from $13-53 \%(p$-value $<0.001)$. 
In terms of oxygen receiving status, of those children having hypoxemia $(\mathrm{SpO} 2<90)$ at diagnosis or any point during admission, the result showed that the majority (95\%) of them received oxygen. However, it was only $63 \%$ of them were able to receive oxygen at the commencement of the project. This shows that children with hypoxemia were more or less properly managed. The result was also statistically significant with ( $p$-value< 0.001), Fig. 5.

\section{Discussions}

The finding of the study documented that the functional availability of oxygen has shown a statistically significant increase in Pediatric In-patient Department of general and referral hospitals. Similarly, the functional availability of Pulse Oximetry has shown statistically significant increased. Moreover, clinical practice, SpO2 measurement at diagnosis and admission has also improved.

Oxygen, as an essential drug, should always be available either with a cylinder or concentrator at all critical points of care. It is a medical therapy that saves the lives of many for more than a century(20). A study that assesses health workers perception on the barriers of oxygen therapy for pediatric patients documented that malfunctioning oxygen cylinders and concentrators, limited or no access to pulse oximetry and lack of continued professional training were key barriers to the delivery of oxygen therapy (21). To ensure the availability of medical oxygen, technical and capacity buildings trainings were provided to health workers and biomedical engineers/technicians in selected public hospitals. This initiative yields promising results by contributing to the country's effort in the reduction of deaths due to pneumonia by increasing access and availability of oxygen. This is witnessed by the functional availability of oxygen, which has shown a statistically significant increase from $62 \%$ at baseline (December 2015)(19) to 100\% in December 2019 at the PIPD of general and referral hospitals ( $p$-value < 0.001). Moreover, beyond enhancing the availability of medical oxygen, the overall medical oxygen practice such as prescription rate (72-91\%), prescription that states flow rate (13-53\%), oxygen provision (63-95\%) practice have also improved as a result of the interventions of the project.

Pulse oximetry (POx) is a non-invasive device that is used to measure oxygen saturation level in the blood as a diagnosis of hypoxemia (22). As a standard clinical practice POx should be applied to all admitted children at least once during admission and for all children with pneumonia. A study reported that the availability of oximetry has increased the referral rate for severely hypoxemic children with statistically significant result(23). Cognizant of the importance of POx, training and regular technical supports were provided to increase access and availability of POx in selected public hospitals. As a result, the functional availability of POx increased from 45\% at baseline (December 2015)(19) to $96 \%$ in December 2019 at PIPD of general and referral hospitals. The increase was also statistically significant ( $p$-value $<0.001)$.

Various studies suggest that regular technical support and supportive supervision is one of the key tasks in the management of the health system which is also pertinent to improve the delivery of quality services (24). More importantly, in low-resource settings, supportive supervisions are pivotal in enhancing the motivation and performance of health workers in delivering quality services $(25,26)$. Moreover, another 
study verified that the introduction of supportive supervision as part of capacity building and service improvement initiatives in countries like Bangladesh, Brazil, Honduras, Kenya, Nepal, and Tanzania, which have registered promising results in both service quality and providers' performance (24).

Likewise, our implementation strategies and the results achieved were in line with these. Various researchers suggested that capacity building with regular support can increase both the performance and motivation of health workers which in turn ultimately improves the accessibility and quality of service delivery $(24,27,28)$. A systematic review also corroborated that the association between clinical supervision of health workers and the effectiveness of care(28). The review reported that substantial improvement in the process and compliance of care associated with enhanced patient health outcomes(28). This is also in agreement with our results that demonstrated the improvement in the process of oxygen therapy (such as oxygen prescription, flow rate and oxygen receiving status) provision for pediatric patients.

As our findings documented that capacity building and regular supportive supervisions were made throughout the project life; this enhances the availability and functionality of medical oxygen and pulse oximetry in selected public hospitals. This is substantiated by a recent study that assesses the frequency of supportive supervision and its dose-response relationship. The study found that an optimum number of regular supportive supervision has an incremental effect on service delivery improvements(29).

Furthermore, research findings also reported that the technical support and supervision of government biomedical departments strongly facilitated equipment maintenance which substantially augments the availability of medical oxygen which in turn ultimately enhances quality service provision (20) (30). In line with this, the project in collaboration with national and regional health bureaus trained and provided regular technical support to biomedical engineers/technicians which contribute to the availability of medical oxygen and increase service quality in health facilities.

\section{Limitation Of The Study}

Although the selected hospitals were across all the tiers of the health system and represent all regions and city administrations the hospitals were not selected randomly. Besides, the sample size did not also follow rigorous sampling and sample size determination procedures. However, almost all hospitals across each tier and regions have more or less similar settings. Therefore, our findings can be extrapolated for the rest of public hospitals in Ethiopia and to other contexts with similar set-ups.

\section{Conclusions}

Based on the intervention results, we can conclude that multifaceted approaches targeting health care workers capacity, increased device procurement and on-site device maintenance with on-site mentorship can improve the availability of medical oxygen and pulse oximetry and clinical practice of oxygen therapy in health facilities. Therefore, ensuring device availability along with regular technical support and close 
follow-up of healthcare workers and facilities are key and the initiative needs to be scaled-up to bring a large scale improvement and change.

\section{Abbreviations}

BME/Ts: Biomedical Engineers/Technicians; EPHI: Ethiopian Public Health Institute; EPSA: Ethiopian Pharmaceutical Supply Agency; HCWs: Health Care Workers; MoH: Ministry of Health; NICU: Newborn Intensive Care Unit; PIPD: Pediatric Inpatient Departments; POx: Pulse Oximetry; RHBs: Regional Health Bureaus;

\section{Declarations}

\section{Ethics approval and consent to participate}

The research protocol was reviewed and ethical clearance obtained from the Ethiopian Public Health Institute (EPHI)[1] with protocol number EPHI-IRB-175-2019, with letter of approval dated on 19 June 2019 and referenced EPHI13.6/136. Besides, letter of confirmation/clearance was also obtained from Ministry of Health (MoH) and Regional Health Bureaus (RHBs). Oral consent was obtained from all research participants. The study had no known risk and no payment was made to participants.

\section{Consent for publication}

Not applicable

\section{Availability of data and materials}

The datasets used and/or analyzed during the current study are available from the corresponding author on reasonable request.

\section{Competing interests}

The authors declare that the research was conducted in the absence of any commercial or financial relationships that could be interpreted as a potential conflict of interest.

Funding: Accelerating Access to lifesaving child Health Commodities and Services in Ethiopia is a Bill \& Melinda Gates Foundation funded Child Survival program under cooperative agreement Investment ID: INV-009817 / OPP1133423. The program is implemented by the Clinton Health Access Initiative in collaboration with local Government. The funder does not have any role in the design of this study, data collection, analysis and writing of the manuscript.

\section{Authors' contributions}

'HST', 'YAA', 'AYD', 'DBW', 'ZFF', 'AB', 'ABB', 'FL' 
All authors equally made a substantial contribution to conceiving and designing the study, responsible for overseeing the fieldwork, cleaning the data, analyzing the data, interpreting the analysis and drafting the manuscript. All authors read the final document and approved it. HST, the corresponding author, submitted the manuscript for publication.

\section{Acknowledgements}

The investigators are indebted to all participants for their cooperation during data collection. This study, availability of medical oxygen and pulse oximetry and clinical practice of oxygen therapy, is made possible by the generous support of the Bill \& Melinda Gates Foundation.

\section{Authors' information}

HST: MD, MSc. Senior Program Manager at Clinton Health Access Initiative-Child Survival Program/Essential Medicine, Addis Ababa, Ethiopia,

YAA: MD, Country Director at Clinton Health Access Initiative- Addis Ababa, Ethiopia,

AYD: MPH, MSc. M\&E Office at Clinton Health Access Initiative- Child Survival Program/Essential Medicine, Addis Ababa, Ethiopia,

DBW: MPH, Program Manager at Clinton Health Access Initiative- Child Survival Program/Essential Medicine, Addis Ababa, Ethiopia,

ZFF: MPH, M\&E Analyst at Clinton Health Access Initiative- Child Survival Program/Essential Medicine, Addis Ababa, Ethiopia,

ABB: MPH, Program Coordinator at Clinton Health Access Initiative- Child Survival Program/Essential Medicine, Addis Ababa, Ethiopia,

AB: MA, Global Director at Clinton Health Access Initiative- SRMNCH - Global Essential Medicines, Boston, USA,

FL: MPH, Global Associate Director at Clinton Health Access Initiative-SRMNCH - Global Essential Medicines, Boston, USA

[1] EPHI is the national Institutional Review Boards (IRB's) through its Scientific and Ethical Review Office (SERO), in charge of ethical review and approval of health and nutrition related researches.

\section{References}

1. Duke T, Graham SM, Cherian MN, Ginsburg AS, English M, Howie S, et al. Oxygen is an essential medicine: A call for international action. Int J Tuberc Lung Dis. 2010;14(11):1362-8. 
2. Subhi R, Adamson M, Campbell H, Weber M, Smith K, Duke T. The prevalence of hypoxaemia among ill children in developing countries: a systematic review. Lancet Infect Dis. 2009 Apr;9(4):219-27.

3. UNICEF Data. Monitoring the situation of children and women.

4. Pneumonia Progress R. 2020; https://www.jhsph.edu/ivac/wpcontent/uploads/2020/11/IVAC_PDPR_2020.pdf.

5. World Pneumonia Day. WHO Fact Sheet. 2020; https://stoppneumonia.org/latest/world-pneumoniaday/.

6. Herbert LJ, Wilson IH. Pulse oximetry in low-resource settings. Breathe. 2012;9(2):90-7.

7. World Health Organization. Oxygen therapy for children. Oxyg Ther Child a Man Heal Work [Internet]. 2016;57. Available from:

http://apps.who.int/iris/bitstream/10665/204584/1/9789241549554_eng.pdf.

8. Abuka T. Prevalence of pneumonia and factors associated among children 2-59 months old in Wondo Genet district, Sidama zone, SNNPR, Ethiopia. Curr Pediatr Res [Internet]. 2017;21(1):19-25. Available from: www.currentpediatrics.com.

9. United Nations Children's Fund. Pneumonia in Children Under Five - UNICEF Data. Unicef [Internet]. 2018;1. Available from: http://dx.plos.org/10.1371/journal.pmed.1001421.

10. Ginsburg AS, Van cleve WC, Thompson MIW, English M. Oxygen and pulse oximetry in childhood pneumonia: A survey of healthcare providers in resource-limited settings. J Trop Pediatr. 2012;58(5):389-93.

11. Usen S, Weber M, Mulholland K, Jaffar S, Oparaugo A, Omosigho C, et al. Clinical predictors of hypoxaemia in Gambian children with acute lower respiratory tract infection: prospective cohort study. Bmj. 1999;318(7176):86-91.

12. Fouzas S, Politis P, Skylogianni E, Syriopoulou T, Priftis KN, Chatzimichael A, et al. Knowledge on Pulse Oximetry Among Pediatric Health Care Professionals: A Multicenter Survey. Pediatrics [Internet]. 2010;126(3):e657-62. Available from:

http://pediatrics.aappublications.org/cgi/doi/10.1542/peds.2010-0849.

13. Marzia Lazzerini N, Seward N, Lufesi R, Banda S, Sinyeka G, Masache. Bejoy Nambiar, Charles Makwenda, Anthony Costello EDM. HHS Public Access. Lancet Glob Heal. 2017;106(3):201-7.

14. Saha S, Hasan M, Kim L, Farrar JL, Hossain B, Islam M, et al. Epidemiology and risk factors for pneumonia severity and mortality in Bangladeshi children $<5$ years of age before 10-valent pneumococcal conjugate vaccine introduction. BMC Public Health [Internet]. 2016;16(1):1-12. Available from: http://dx.doi.org/10.1186/s12889-016-3897-9.

15. Subhi R, Adamson M, Campbell H, Weber M, Smith K, Duke T. The prevalence of hypoxaemia among ill children in developing countries: a systematic review. Lancet Infect Dis [Internet]. 2009;9(4):21927. Available from: http://dx.doi.org/10.1016/S1473-3099(09)70071-4.

16. Walsh BK, Smallwood CD. Pediatric Oxygen Therapy: A Review and Update. Respir Care [Internet]. 2017;62(6):645-61. Available from: http://rc.rcjournal.com/lookup/doi/10.4187/respcare.05245. 
17. by Dove Press published. COPD-103607-acute-oxygen-therapy-prescribing-practices-a-review-of-the-. 2016;1067-75.

18. Child. Survival program Training Database. (program document).

19. Accelerating Policy Change. Translation and Implementation for Pneumonia and Diarrhea Commodities in Ethiopia. Report. 2016; (program document).

20. Bakare AA, Graham H, Ayede Al, Peel D, Olatinwo O, Oyewole OB, et al. Providing oxygen to children and newborns: A multi-faceted technical and clinical assessment of oxygen access and oxygen use in secondary-level hospitals in southwest Nigeria. Int Health. 2019;12(1):60-8.

21. Dauncey JW, Olupot-Olupot P, Maitland K. Healthcare-provider perceptions of barriers to oxygen therapy for paediatric patients in three government-funded eastern Ugandan hospitals; A qualitative study. BMC Health Serv Res. 2019;19(1):1-9.

22. Burn SL, Chilton PJ, Gawande AA, Lilford RJ. Peri-operative pulse oximetry in low-income countries: a cost-effectiveness analysis. Bull World Health Organ. 2014;92(12):858-67.

23. McCollum ED, King C, Deula R, Zadutsa B, Mankhambo L, Nambiar B, et al. Pulse oximetry for children with pneumonia treated as outpatients in rural Malawi. Bull World Health Organ. 2016;94(12):893-902.

24. Purity M, Eilish M, Ogenna U, Honorati M, Henry M. The Impact of Supportive Supervision on the Implementation of HRM processes; A Mixed-Methods study in Tanzania. Heal Syst Policy Res. 2017;04(01):1-9.

25. Hill Z, Dumbaugh M, Benton L, Källander K, Strachan D, ten Asbroek A, et al. Supervising community health workers in low-income countries - a review of impact and implementation issues. Glob Health Action. 2014;7(1).

26. Avortri GS, Nabukalu JB, Nabyonga-Orem J. Supportive supervision to improve service delivery in low-income countries: is there a conceptual problem or a strategy problem? BMJ Glob Heal. 2019;4(Suppl 9):e001151.

27. Roberton T, Applegate J, Lefevre AE, Mosha I, Cooper CM, Silverman M, et al. Initial experiences and innovations in supervising community health workers for maternal, newborn, and child health in Morogoro region, Tanzania. Hum Resour Health [Internet]. 2015;13(1):1-12. Available from: http://dx.doi.org/10.1186/s12960-015-0010-x.

28. Snowdon DA, Leggat SG, Taylor NF. Does clinical supervision of healthcare professionals improve effectiveness of care and patient experience? A systematic review. BMC Health Serv Res. 2017;17(1):1-11.

29. Desta BF, Beshir IA, Tefera BB, Argaw MD, Demeke HZ, Kibret MA. Does frequency of supportive supervisory visits influence health service delivery?-Dose and response study. PLoS One [Internet]. 2020;15(6):1-13. Available from: http://dx.doi.org/10.1371/journal.pone.0234819.

30. Bradley BD, Chow S, Nyassi E, Cheng Y-L, Peel D, Howie SRC. A retrospective analysis of oxygen concentrator maintenance needs and costs in a low-resource setting: experience from The Gambia. 
Health Technol (Berl) [Internet]. 2015;4(4):319-28. Available from: https://doi.org/10.1007/s12553015-0094-2.

\section{Figures}

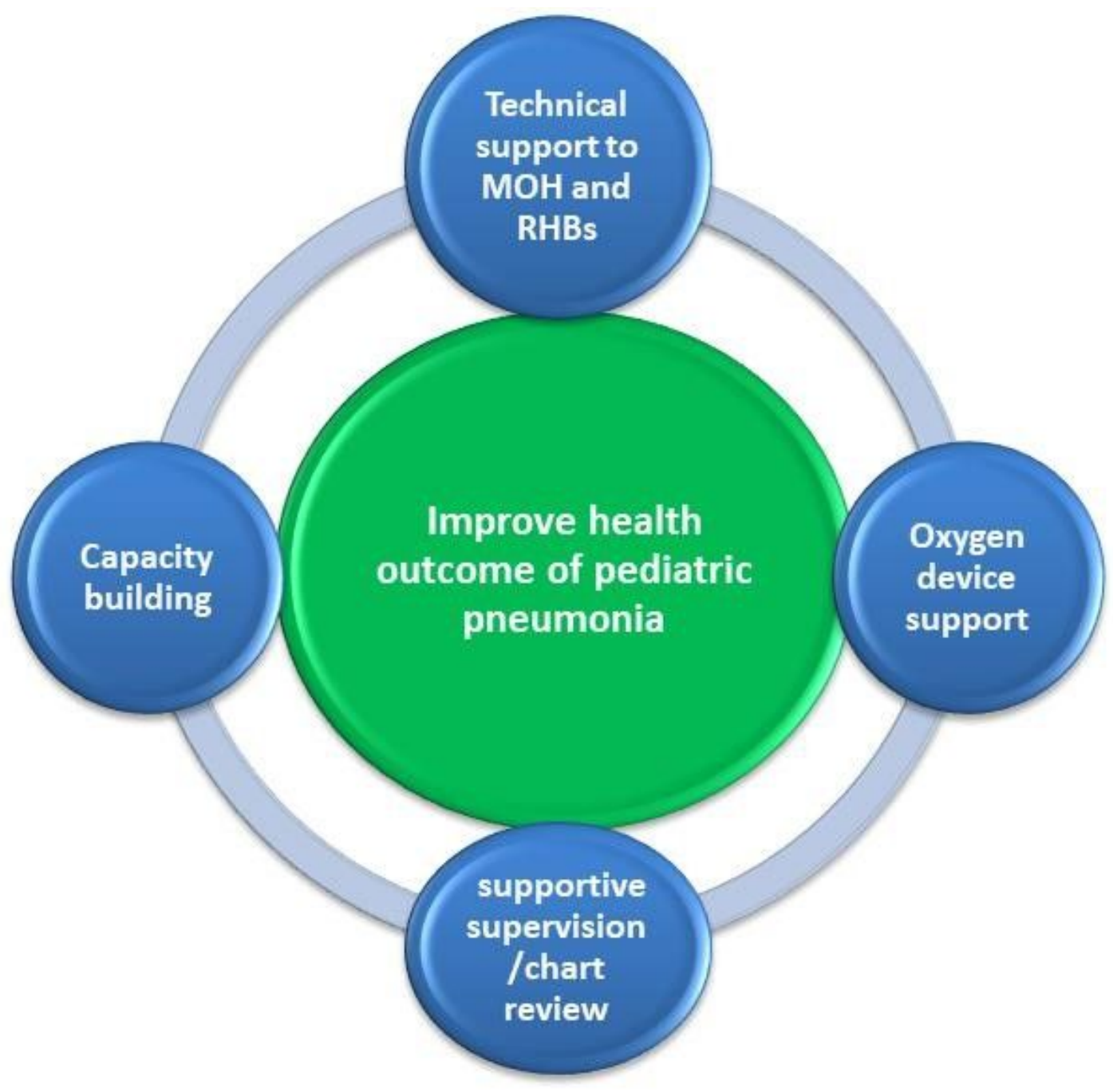

\section{Figure 1}

Intervention frameworks (the interventions are contributing towards improving health outcomes of pediatric pneumonia) 


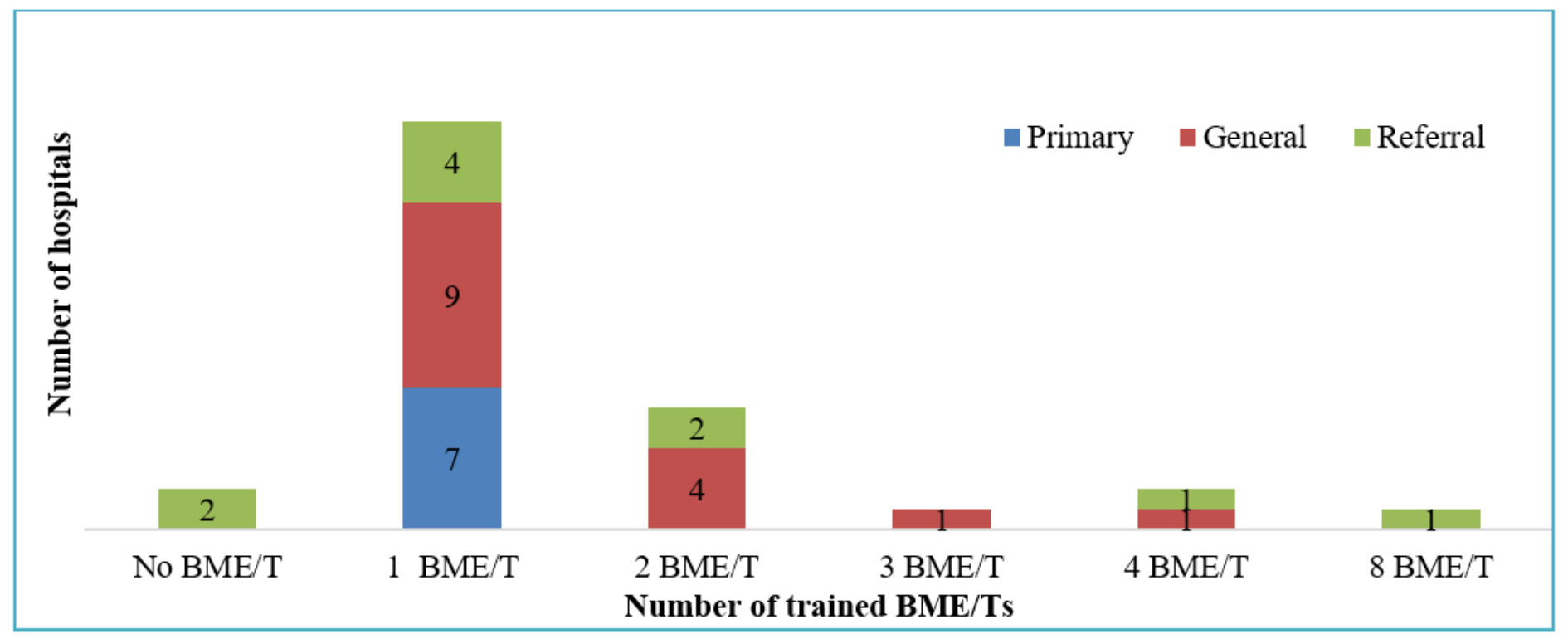

\section{Figure 2}

Number of trained BME/Ts on concentrator and POx maintenance by types of hospitals

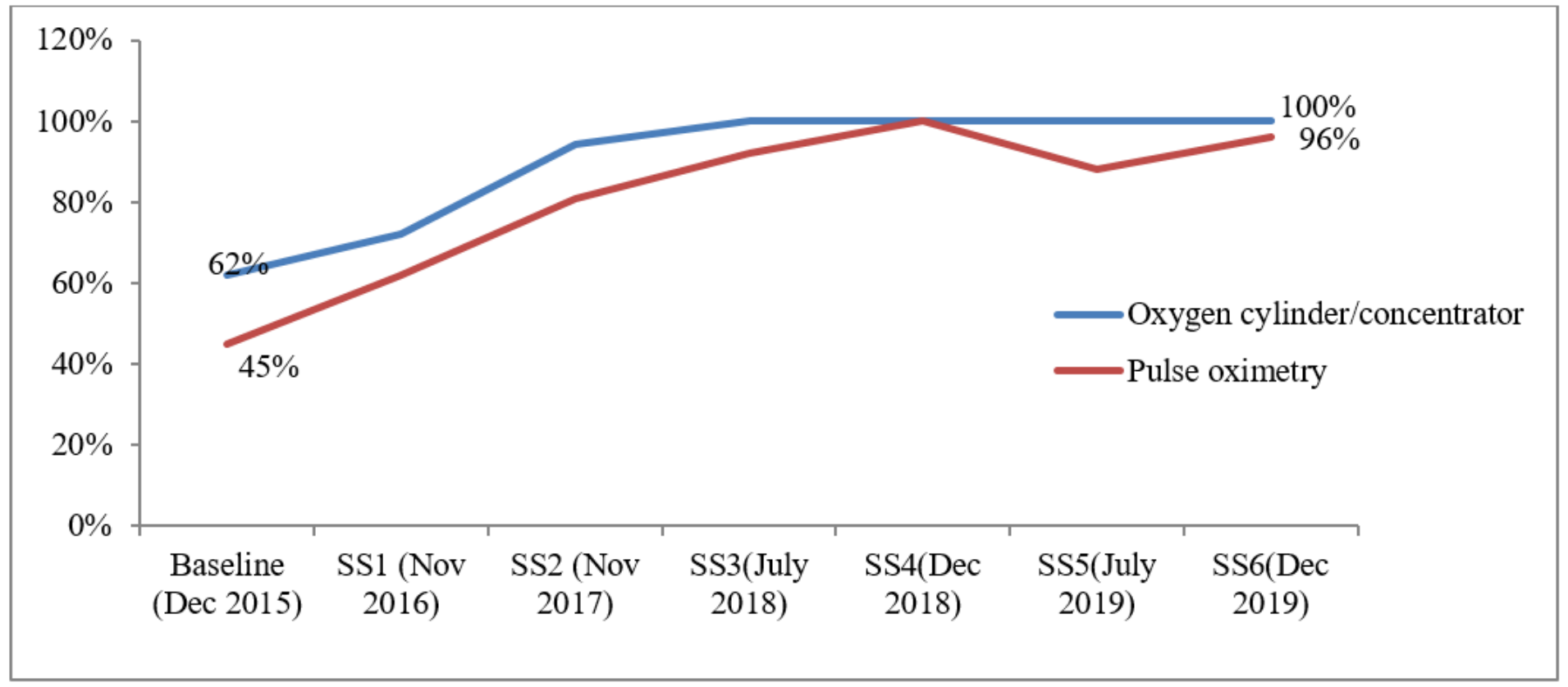

\section{Figure 3}

Trends in the availability of functional oxygen and POx at PIPD in general and referral hospitals 


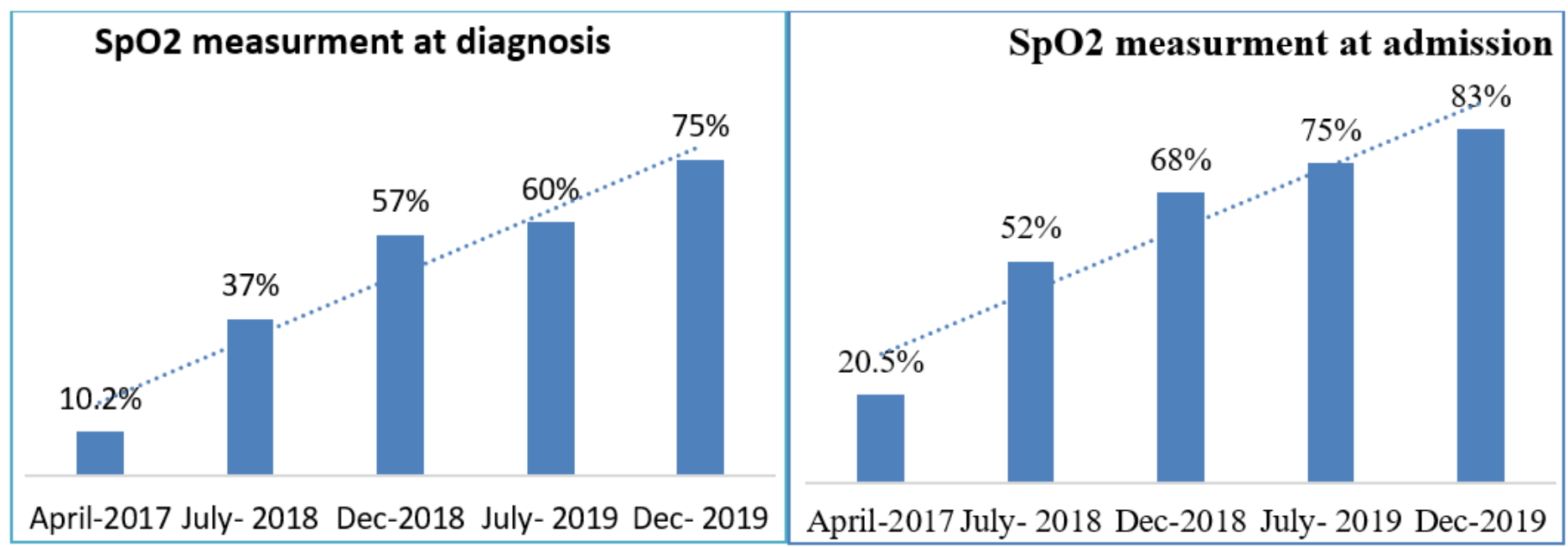

Figure 4

Status of POx assessment at diagnosis and admission

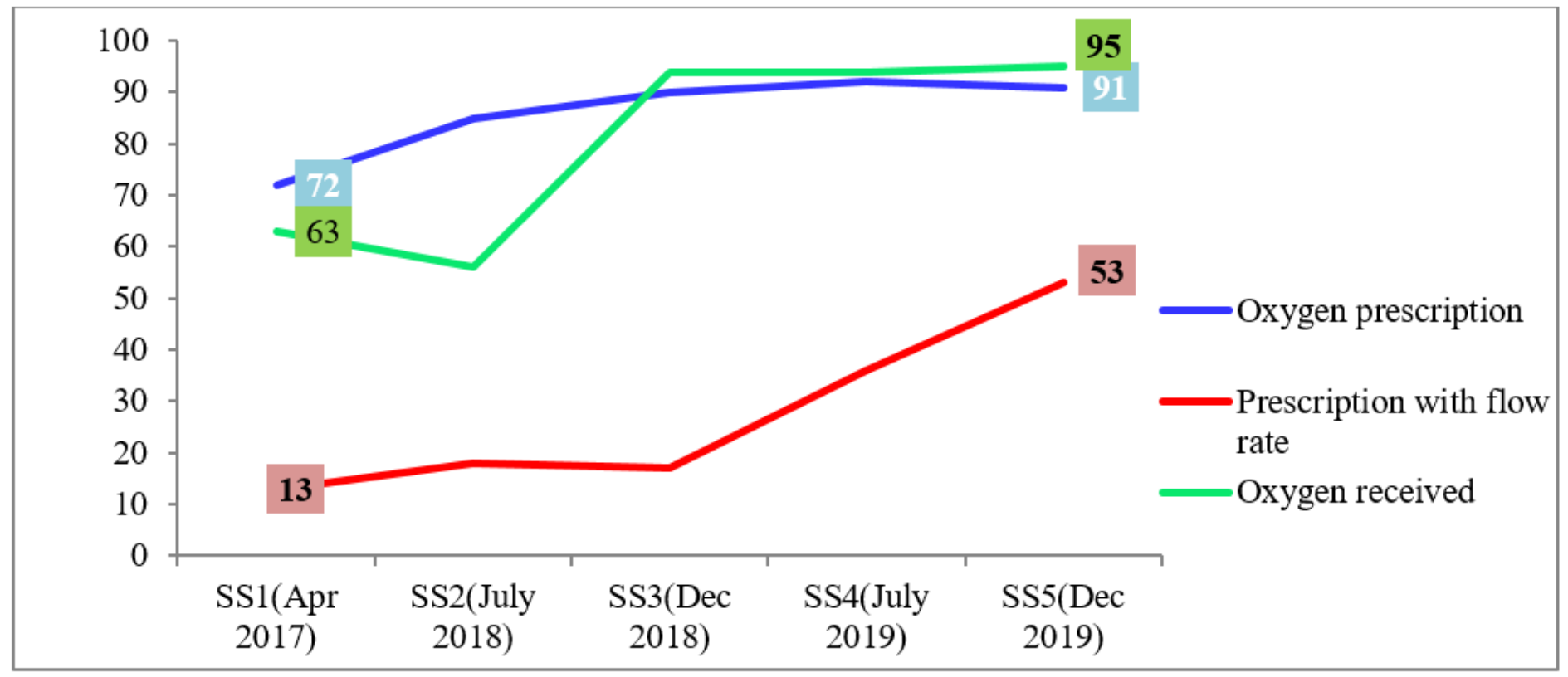

Figure 5

Trends in oxygen prescription, prescription with flow rate and oxygen received

\section{Supplementary Files}

This is a list of supplementary files associated with this preprint. Click to download.

- Additionalfile1SupportiveSupervisionTool.docx

- STROBEChecklist.docx 